\title{
Breakup following interactions with light targets: Investigating new methods to probe nuclear physics input to the cosmological lithium problem
}

\author{
K.J. Cook ${ }^{1, a}$, D.H. Luong ${ }^{1}$, I.P Carter ${ }^{1}$, M. Dasgupta ${ }^{1}$, D.J. Hinde ${ }^{1}$, S. McNeil ${ }^{1}$, D. Rafferty ${ }^{1}$, K. Ramachandran ${ }^{2}$, C. \\ Simenel ${ }^{1}$, and E. Williams ${ }^{1}$ \\ ${ }^{1}$ Department of Nuclear Physics, Research School of Physics and Engineering, The Australian National University, Canberra, \\ ACT 0200, Australia \\ ${ }^{2}$ Nuclear Physics Division, Bhabha Atomic Research Centre, Trombay, Mumbai-400085, India
}

\begin{abstract}
A well known issue with concordance cosmology is the cosmological lithium problem, where models of Big Bang Nucleosynthesis indicate abundances of ${ }^{7} \mathrm{Li}$ three to four times larger than values inferred via spectroscopic measurements of metal-poor halo stars [1]. Since the source of this discrepancy remains unclear [2], it is vital to fully understand the nuclear reactions that affect the production of ${ }^{7} \mathrm{Li}$ during the Big Bang [3]. At the Australian National University, experimental equipment and analysis techniques have been developed for nuclear reaction studies at energies near the fusion barrier, exploiting large solid angle detectors to enable the investigation of breakup without a priori assumption of the breakup kinematics. The extension to reactions of astrophysical interest may help shed light on these reactions. Recent experiments, using these new techniques, have provided a complete picture of the breakup mechanisms of light nuclei in collisions with heavy targets [4]. The present work focuses on obtaining a complete picture of breakup mechanisms of ${ }^{7} \mathrm{Li}$ following interactions with ${ }^{27} \mathrm{Al}$. It has been found that breakup is almost exclusively triggered by nucleon transfer between the colliding partners, to a larger extent than was found for heavier targets. The findings of these experiments, as well as progress towards extensions to astrophysically relevant reactions, such as $\mathrm{d}+{ }^{7} \mathrm{Be}[5]$ will be presented.
\end{abstract}

\section{Introduction}

The significant discrepancy between predicted and inferred primordial abundances of ${ }^{7} \mathrm{Li}$ is an outstanding problem in concordance models of the Big Bang, with abundances predicted from models of Big Bang Nucleosynthesis $(\mathrm{BBN})$ three to four times higher than those inferred from spectroscopic observations of metal-poor halo stars $[1,2]$. The discrepancy, which has been well established since 1982 [6], is yet to find a satisfactory solution, although there has been significant effort to achieve one. Proposed solutions for the primordial lithium problem can be found in many areas of nuclear astrophysics, which include but are by no means limited to: stellar models with increased turbulence and diffusion between surface and burning layers [7]; inferring primordial abundances from low metallicity gases in the Small Magellanic Cloud [8]; non-standard cosmologies e.g.[9]; physics beyond the standard model e.g. [10]; and nuclear physics e.g.[3]. It is nuclear physics solutions to the primordial lithium problem that are the focus of the present work.

Nuclear solutions center around the re-examination of the nuclear reactions that contribute to the abundances of ${ }^{7} \mathrm{Li}$ produced in models of BBN. Shown in Figure 1 is a

\footnotetext{
ae-mail: kaitlin.cook@anu.edu.au
}

simplified nuclear reaction network, showing the dominant reactions that contribute to ${ }^{7} \mathrm{Li}$ abundances. Crucially, the dominant process forming ${ }^{7} \mathrm{Li}$ during $\mathrm{BBN}$ is not through the direct $\left.{ }^{3} \mathrm{H}(\alpha, \gamma)\right)^{7} \mathrm{Li}$ fusion reaction, but through the production of ${ }^{7} \mathrm{Be}$, and its subsequent decay through electron capture with a half life of 52.3 days. Therefore, the search for nuclear physics solutions to the primordial lithium problem are, in the main part, focused on reactions that alter the abundance of ${ }^{7} \mathrm{Be}$. The ${ }^{3} \mathrm{He}(\alpha, \gamma){ }^{7} \mathrm{Be}$ production reaction rate is strongly constrained by observations of stellar neutrino production. As such, a variation of the production reaction rate cannot solve the ${ }^{7} \mathrm{Li}$ problem without resulting in tension with neutrino observations. However, reactions that destroy ${ }^{7} \mathrm{Be}$ without producing ${ }^{7} \mathrm{Li}$ are candidates for nuclear physics solutions to the primordial lithium problem.

\subsection{The ${ }^{7} \mathrm{Be}(\mathrm{d}, \mathrm{p}) 2 \alpha$ reaction}

Candidate reactions that may relieve the primordial lithium problem require (a) cross-sections that are sufficiently large at BBN energies, and (b) that the reactants are sufficiently abundant during BBN. One such candidate reaction is ${ }^{7} \mathrm{Be}(\mathrm{d}, \mathrm{p}) 2 \alpha$, which has been shown through a sensitivity study [11] to be able to resolve the ${ }^{7} \mathrm{Li}$ problem if the reaction rate is a factor of 100 times larger than the initial estimate [12]. Instead, a new measurement [5] found a 


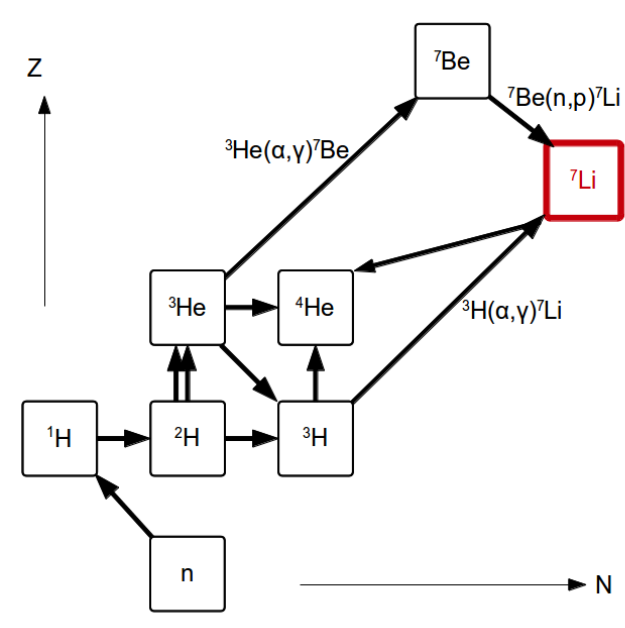

Figure 1. Simplified reaction network showing reactions forming ${ }^{7} \mathrm{Li}$ during the Big Bang. Adapted from [2].

reaction rate 10 times smaller than the earlier value. However, this measurement was insufficiently sensitive to excitations of ${ }^{8} \mathrm{Be}$ above the first excited state, and it is thus impossible to be confident in the measured reaction rate. Further theoretical work is continuing in this area [3]. Experimentally, it is imperative that the ${ }^{7} \mathrm{Be}(\mathrm{d}, \mathrm{p}) 2 \alpha$ reaction rate is re-examined, with a focus on excited states of ${ }^{8} \mathrm{Be}$, so that its status as a candidate reaction for resolving the primordial lithium problem may be fully understood. This can be achieved through the measurement of $\alpha$ particles in coincidence, rather than the low energy protons produced when ${ }^{8} \mathrm{Be}$ is populated above the ground-state resonance. A new detector array developed at the ANU enables us to do just that.

\section{The Breakup Array for Light Nuclei}

The Breakup Array for Light Nuclei (BALiN) is composed of four, $400 \mu \mathrm{m}$ thick, wedge shaped double sided silicon strip detectors (DSSDs) $)^{1}$, angled at $45^{\circ}$ relative to the beam axis, as shown in figure 2(a). With 16 arcs on the front face of each detector and 8 sectors on the back, position information with an effective pixelation of 512 pixels is obtained, and a typical angular coverage is shown in figure 2(b). The large angular coverage, along with the energy and time-of-flight of particles detected in coincidence, allow kinematic reconstruction of breakup events, and thus insights into breakup mechanisms are obtained.

Two of the key breakup observables are (a) the reaction Q-value which gives the energy released or required in the reaction, as well as the state of the target-like recoil nucleus, and (b) the relative energy, $E_{r e l}$, of the breakup fragments. The latter observable gives information related to the state of the projectile-like nucleus and is sensitive to the timescale of the breakup processes. These two

\footnotetext{
${ }^{1}$ Micron Semiconductor Limited, Sussex, UK
}

variables provide fairly complete knowledge of breakup mechanisms. The BALiN array and the kinematic reconstruction methods have been previously successfully implemented to study the breakup of the light nuclei ${ }^{6,7} \mathrm{Li}$ and ${ }^{9} \mathrm{Be}$ on various heavy targets $[4,13]$. These studies have shown that prompt breakup (with timescales on the order of the collision timescale $\sim 10^{-21} \mathrm{~s}$ ) is dominantly triggered by transfer. That is, rather than breakup of ${ }^{7} \mathrm{Li}$ into its cluster constituents $\left({ }^{7} \mathrm{Li} \rightarrow \alpha+t\right)$, the dominant mechanism for prompt breakup on heavy targets is proton pickup forming ${ }^{8} \mathrm{Be}$, which subsequently breaks up into two $\alpha$ fragments.

As these measurements have been made on very heavy targets, far away from the mass of deuterium, it is necessary to examine trends of breakup and the performance of the BALiN array and reconstruction techniques with decreasing target mass. Towards this end, measurements have been performed to examine the breakup in the interaction of ${ }^{7} \mathrm{Li}$ with ${ }^{27} \mathrm{Al}$

\section{Towards ${ }^{7} \mathrm{Be}(\mathrm{d}, \mathrm{p}) 2 \alpha$ : Breakup in the interaction of ${ }^{7} \mathrm{Li}$ with ${ }^{27} \mathrm{Al}$}

Beams of ${ }^{7} \mathrm{Li}$ were delivered by the 14UD pelletron at the Australian National University Heavy Ion Accelerator Facility onto an ${ }^{27} \mathrm{Al}$ target at a below-barrier energy of 7.5 $\mathrm{MeV}$. When two or more particles were incident on the array in coincidence, their energy, position, and time-offlight were recorded. By examining the time-of-flight and the energy of each particle, particles of different masses may be separated through the relation

$$
t=d \sqrt{\frac{m}{2 E}},
$$

where $d$ is the detector-target distance, $m$ the mass of the particle, and $E$ the measured energy. This allowed the identification of the breakup fragments as pairs of either $\alpha+\alpha$ or $\alpha+p$ (no other breakup modes e.g. $\alpha+d$ or $\alpha+t$ were observed). The energies and angles of each particle were then used to reconstruct the Q-value and relative energy of each event, by making use of momentum conservation. Shown in figure 3(a) is the reconstructed Q-value against $E_{r e l}$ for all events, for the two breakup modes observed. Shown in blue are breakup events resulting from one proton pickup: ${ }^{8} \mathrm{Be}$ $\rightarrow \alpha+\alpha$, and in purple are breakup events resulting from two neutron stripping: ${ }^{5} \mathrm{Li} \rightarrow \alpha+p$. Horizontal bands are immediately apparent, each one corresponding to interactions populating a particular state of the target-like recoil nucleus and a range of relative energies.

The relative populations of the ground and excited states of ${ }^{26} \mathrm{Mg}$ formed following one proton pickup is seen through the reconstructed Q-value spectrum shown in figure 3(b), while the peaks in the second Q-value spectrum (figure 3(c)) show the population of the ground and excited states of ${ }^{29} \mathrm{Al}$ formed through two neutron 


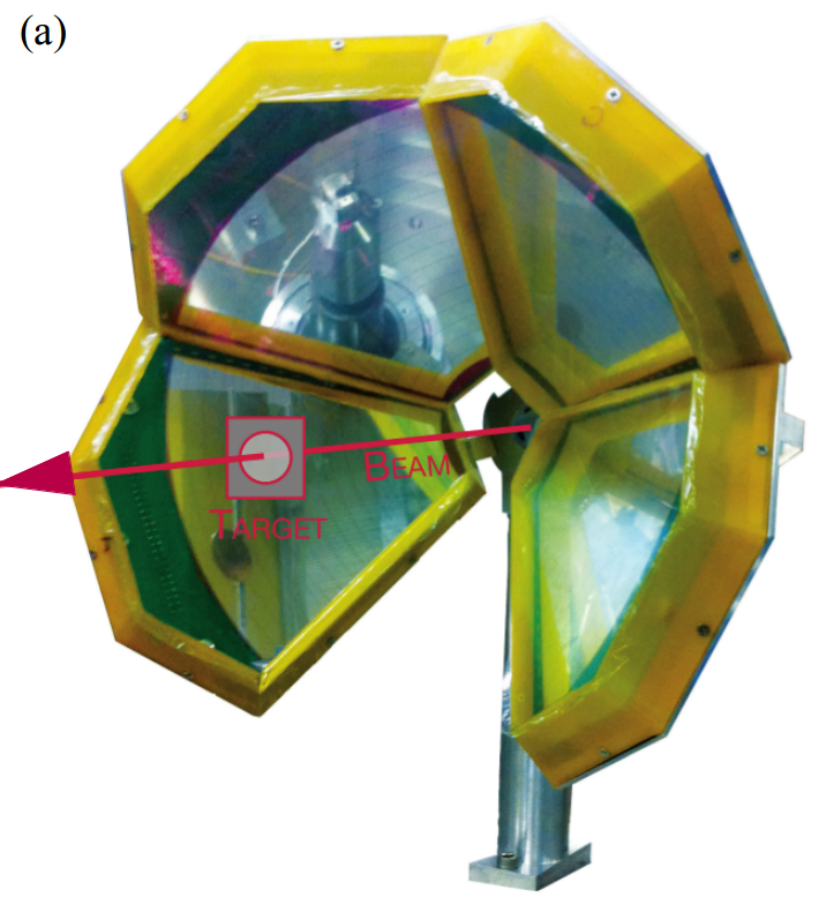

(b)

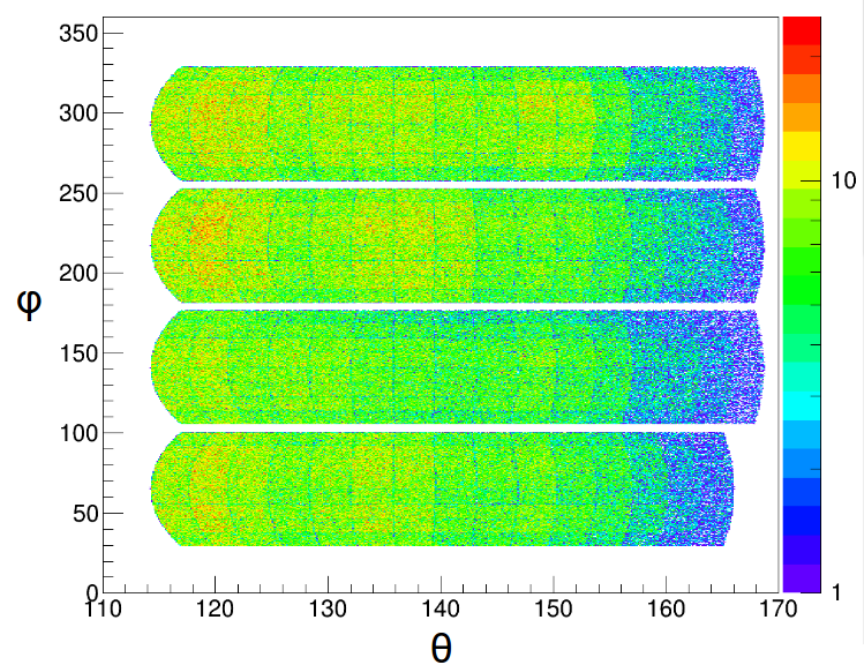

Figure 2. (a) The Breakup Array for Light Nuclei, composed of four DSSDs mounted on a central hub at an angle of $45^{\circ}$ with respect to their bisector, towards the focal point of the hub. Also shown is the target position, and the direction of the beam (arrow). (b) The angular coverage of the array's 512 pixels, shown in scattering angle $\theta$ and azimuthal angle $\phi$ for the measurement of the breakup of ${ }^{7} \mathrm{Li}$ after interaction with ${ }^{27} \mathrm{Al}$.

stripping. Due to the highly positive ground-state Qvalues of each process $(+9.08$ and $+6.20 \mathrm{MeV}$ for one proton pickup and two neutron stripping, respectively), it is perhaps not surprising that these reactions populate ${ }^{26} \mathrm{Mg}$ and ${ }^{29} \mathrm{Al}$ to highly excited states.

While the reconstructed Q-value of these reactions enables an understanding of the state of the target-like-recoil nucleus produced in these transfer induced breakup reactions, the reconstructed $E_{\text {rel }}$ gives valuable information as to the state of the projectile-like nucleus after transfer, as well as the timescale of the reaction. Shown in figure 3(d) is the $E_{r e l}$ spectrum for ${ }^{8} \mathrm{Be} \rightarrow \alpha+\alpha$ breakup. The peak at a relative energy of $92 \mathrm{keV}$ indicates breakup of ${ }^{8} \mathrm{Be}$ from the (relatively long-lived $t_{1 / 2} \sim 10^{-16} \mathrm{~s}$ ) ground-state, far away from the target-like nucleus. Events with larger $E_{r e l}$ are due to breakup of ${ }^{8} \mathrm{Be}$ after it has been populated close to the target-like-nucleus in either the first resonance $\left(J^{\pi}=2^{+}\right)$or a non-resonant state, which have lifetimes on the order of the collision timescale $\left(\sim 10^{-21} \mathrm{~s}\right)$. The proximity to the target-like nucleus results in post-acceleration of the breakup fragments due to the presence of the Coulomb field and thus an increase in the relative energy of the fragments. Unlike the ${ }^{8} \mathrm{Be} \rightarrow \alpha+\alpha$ events, the ${ }^{5} \mathrm{Li} \rightarrow \alpha+p$ events show no sharp peak in $E_{\text {rel }}$. In contrast to ${ }^{8} \mathrm{Be},{ }^{5} \mathrm{Li}$ has no relatively long-lived resonances, and breakup will occur on the collision timescale $\left(\sim 10^{-21} \mathrm{~s}\right)$. As such, all breakup is post-accelerated, resulting in the broad distribution of $E_{\text {rel }}$ seen in figures 3(a) and (d).
Notably absent in the breakup of ${ }^{7} \mathrm{Li}$ after interactions with ${ }^{27} \mathrm{Al}$ is any breakup through the direct channel ${ }^{7} \mathrm{Li}$ $\rightarrow \alpha+t$ or through one neutron stripping forming ${ }^{6} \mathrm{Li}$ $\rightarrow \alpha+d$. This is in contrast to studies on heavy nuclei such as ${ }^{208} \mathrm{~Pb}$, where the direct breakup mode as well as one proton pickup ${ }^{8} \mathrm{Be} \rightarrow \alpha+\alpha$ and one neutron stripping ${ }^{6} \mathrm{Li} \rightarrow \alpha+d$ were observed [4]. It is therefore apparent that the breakup modes of ${ }^{7} \mathrm{Li}$ are strongly dependent on the species of the colliding partner, and therefore the energetics of the reaction.

\section{Conclusions}

Using the ANU BALiN array, an experimental technique has been successfully established to measure the reaction dynamics of breakup of light nuclei after interactions with nuclei with masses ranging from 27 to 208. Future work focuses on extending the experimental methods to measure breakup in astrophysically relevant reactions.

\section{Acknowledgements}

This work was supported by Australian Research Council grants FL110100098, DP130101569 and DP110102879. The authors would also like to thank E. Simpson for the illuminating discussions, and the ANU Department of $\mathrm{Nu}$ clear Physics technical staff, whose expertise and guidance helps make our work possible. 


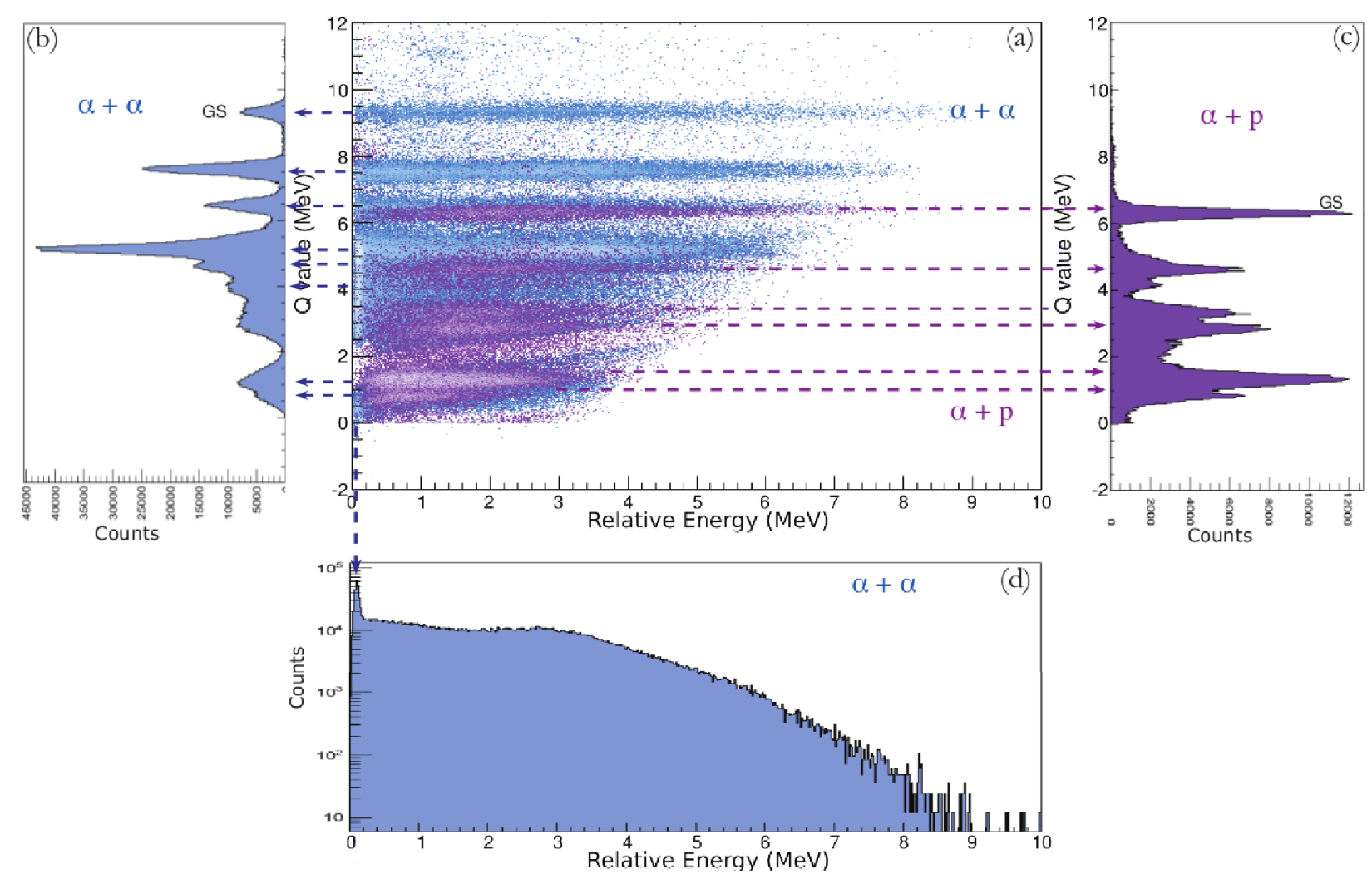

Figure 3. (a) Histogram of relative energy, $E_{\text {rel }}$, against Q-value showing breakup modes in the reaction of ${ }^{7} \mathrm{Li}$ with ${ }^{27} \mathrm{Al}$ at the below barrier energy of 7.5 MeV. Particle identification is achieved through time-of-flight measurement, as described in the text. No evidence of direct ${ }^{7} \mathrm{Li} \rightarrow \alpha+t$ breakup was observed, nor is the one-neutron stripping channel to form ${ }^{6} \mathrm{Li} \rightarrow \alpha+d$. Instead, breakup is triggered by proton pickup to form ${ }^{8} \mathrm{Be} \rightarrow \alpha+\alpha$ (shown in blue), and two neutron stripping to form ${ }^{5} \mathrm{Li} \rightarrow \alpha+p$ (shown in purple). Q-value projections are shown for the $\alpha+\alpha$ and $\alpha+p$ modes in panels (b) and (c) respectively, and the Q-value associated with reactions populating the ground-state of the target-like recoil nucleus is labeled "GS". The $E_{r e l}$ projection for the $\alpha+\alpha$ breakup mode is shown in panel $(\mathrm{d})$.

\section{References}

[1] M. Asplund, D.L. Lambert, P.E. Nissen, F. Primas, V.V Smith, ApJ 644, 229 (2006)

[2] B.D. Fields, Ann. Rev. Nucl. Part. Sci. 61, 47 (2011)

[3] C. Broggini, L. Canton, G. Fiorentini, F.L. Villante, J. Cos. Astro-Particle Phys. 1206, 030 (2012)

[4] D.H. Luong, M. Dasgupta, D.J. Hinde, R. Du Rietz, R. Rafiei, C. Lin, M. Evers, A. Diaz-Torres, Phys. Lett. B 695, 105 (2011)

[5] C. Angulo, E. Casarejos, M. Couder, P. Demaret, P. Leleux, F. Vanderbist, A. Coc, J. Kiener, V. Tatischeff, T. Davinson et al., ApJL 630, 105 (2005)

[6] F. Spite, M. Spite, Astron. Astrophys. 115, 357 (1982)
[7] A.J. Korn, F. Grundahl, O. Richard, P.S. Barklem, L. Mashonkina, R. Collet, N. Piskunov, B. Gustafsson, Nature 442, 657 (2006)

[8] J. C. Howk, N. Lehner, B.D. Fields, G. J. Mathews, Nature 489, 121 (2012)

[9] M. Regis, C. Clarkson, Gen. Rel. Grav. 44, 567 (2012)

[10] A. Coc, J. Uzan, E. Vangioni, Phys. Rev. D 87, 123530 (2013)

[11] A. Coc, E. Vangioni-Flam, P. Descouvemont, A. Adahchour, C. Angulo, ApJ 600, 544, (2004)

[12] P.D. Parker, ApJ 175, 261 (1972)

[13] R. Rafiei, D.H. Luong, D.J. Hinde, M. Dasgupta, R. Du Rietz, Int. J. Mod. Phys. E 20, 835 (2011) 